\title{
Tumefactive Multiple Sclerosis
}

\author{
Satoshi Yamashita, En Kimura, Teruyuki Hirano and Makoto Uchino
}

Key words: tumefactive multiple sclerosis, MRI, MR spectroscopy, brain biopsy

(Inter Med 48: 1113-1114, 2009)

(DOI: 10.2169/internalmedicine.48.2046)
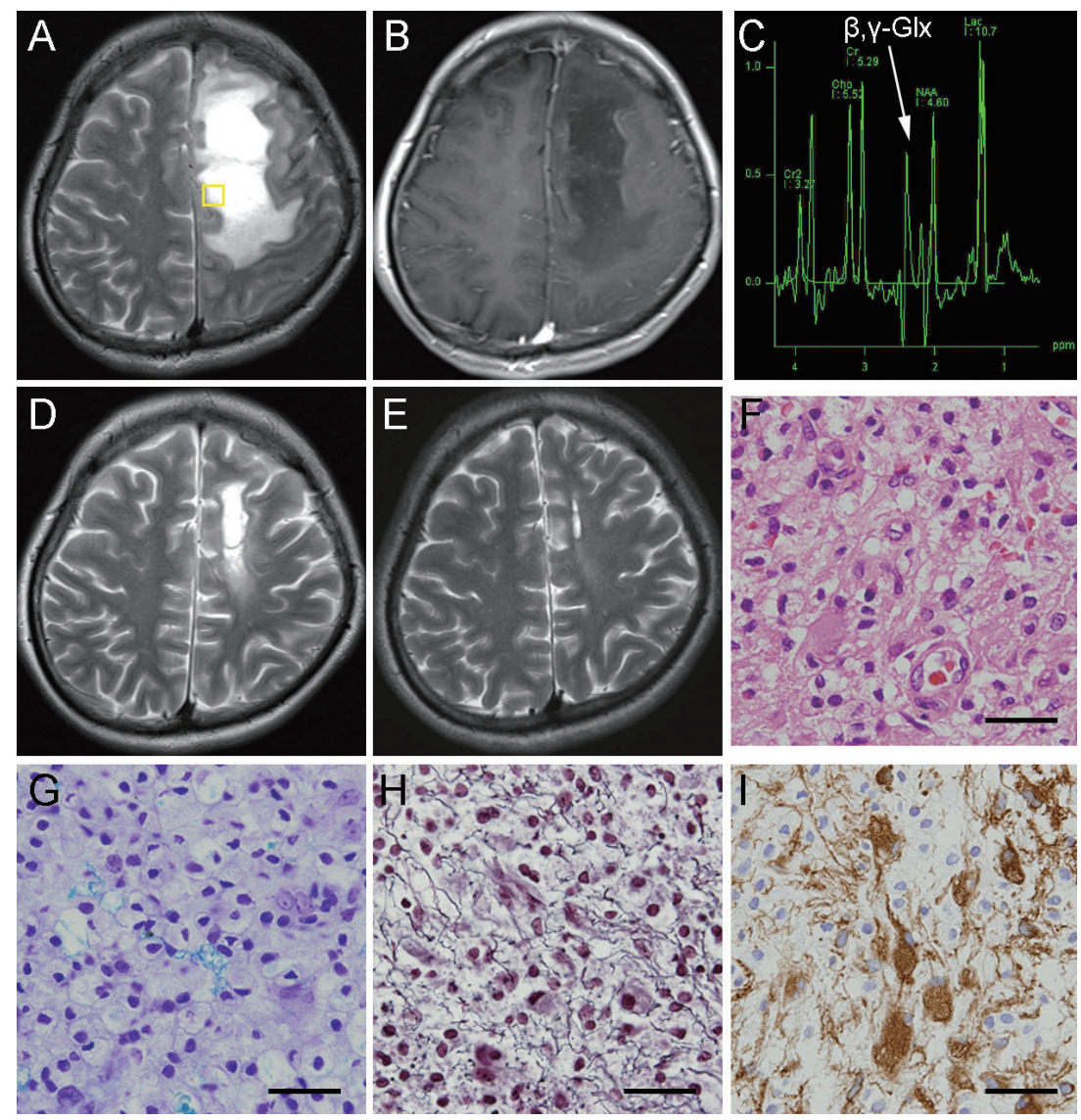

Picture 1. Axial T2-weighted image (A) showed a large mass lesion with surrounding edema and mass effect in the left frontal lobe. An open square indicates voxel localization for MR spectroscopy. T1-weighted image with gadolinium enhancement $(B)$ revealed open ring enhancement. MR spectroscopy (C) showed an abnormal elevation of the glutamate/glutamine ( $\beta, \gamma$-Glx) peaks as well as decreased $\mathrm{N}$-acetylaspartate (NAA) and mild elevation of choline (Cho). Follow-up MRI showed obvious resolution of demyelinating plaque at 1 month (D) and 4 months (E) later. Brain biopsy revealed a macrophage-rich lesion in Hematoxylin and Eosin staining (F) and gliosis of reactive astrocytes in GFAP staining (I). Klüver-Barrera staining (G) demonstrated total myelin loss whereas Bodian staining (H) showed relative axonal preservation. Scale bars, $20 \mu \mathrm{m}$.

We present a rare case of large demyelinating plaque in the brain mimicking intracranial neoplasm. A previously healthy 27-year-old woman noticed clumsiness of her right hand followed by progressive right hemiparesis within sev-

Department of Neurology, Graduate School of Medical Sciences, Kumamoto University, Kumamoto

Received for publication January 12, 2009; Accepted for publication March 17, 2009

Correspondence to Dr. Satoshi Yamashita, y-stsh@pg8.so-net.ne.jp 
eral days. Physical examination revealed severe weakness and hyperreflexia in the right upper and lower extremities. Brain MR imaging disclosed a large ring-enhancing lesion with surrounding edema and mass effect in the left frontal lobe (Pictures 1A, 1B). MR spectroscopy showed an abnormal elevation of the glutamate/glutamine peaks as well as decreased $\mathrm{N}$-acetylaspartate (NAA) and mild elevation of choline (Cho) (Picture 1C). Laboratory findings including routine CSF and IgG index were normal. Myelin basic protein, oligoclonal bands and serum anti-aquaporin 4 antibody were not detected. The patient underwent biopsy of this lesion, and pathology revealed a macrophage-rich lesion with gliosis of reactive astrocytes (Pictures 1F, 1I). KlüverBarrera stain (Picture 1G) demonstrated total myelin loss while Bodian stain (Picture $1 \mathrm{H}$ ) showed relative axonal preservation, consistent with demyelinating disease. There was no evidence of cell neoplasia. She was given a diagnosis of tumefactive multiple sclerosis (MS), and received corticosteroid treatment for acute MS exacerbation. One month later she recovered without any neurological deficit, and 4 months later the demyelinating palque had almost disappeared (Picture 1E). A series of follow-up MRS revealed a decrease of the glutamate/glutamine peaks.

MR spectroscopy has the potential for distinguishing the presence and relative amount of various chemical metabolites in the brain and is therefore a useful technique to further assess various intracranial diseases. However, recent publications have suggested that MR spectroscopy may not be suitable to differentiate MS from neoplasms because of the overlap in decreased NAA/creatine ratio, increased Cho/ creatine ratio, and the variable and nonspecific presence of lactate and lipids in both of these entities (1). A recent report showed that an abnormal elevation of the glutamate/ glutamine peaks is a more critical MR spectroscopy finding in tumefactive lesions of MS in the brain (2), which is in accordance with our finding. These specific findings in MR spectroscopy of tumefactive MS might be helpful to provide a more specific diagnosis without potentially harmful surgery, allowing earlier initiation of the appropriate therapy.

\section{References}

1. Law M, Meltzer DE, Cha S. Spectroscopic magnetic resonance imaging of a tumefactive demyelinating lesion. Neuroradiology 44: 986-989, 2002.

2. Cianfoni A, Niku S, Imbesi SG. Metabolite findings in tumefac- tive demyelinating lesions utilizing short echo time proton magnetic resonance spectroscopy. AJNR Am J Neuroradiol 28: 272277, 2007.

(C) 2009 The Japanese Society of Internal Medicine http://www.naika.or.jp/imindex.html 